\title{
BMJ Open Photopic negative response (PhNR) in the diagnosis and monitoring of raised intracranial pressure in children: a prospective cross-sectional and longitudinal protocol
}

Oliver Rajesh Marmoy (D) , ${ }^{1,2,3}$ Emma Hodson-Tole, ${ }^{4}$ Dorothy Ann Thompson ${ }^{1,3}$

To cite: Marmoy OR, HodsonTole E, Thompson DA. Photopic negative response (PhNR) in the diagnosis and monitoring of raised intracranial pressure in children: a prospective cross-sectional and longitudinal protocol. BMJ Open 2021;11:e047299. doi:10.1136/ bmjopen-2020-047299

- Prepublication history for this paper is available online. To view these files, please visit the journal online (http://dx.doi. org/10.1136/bmjopen-2020047299).

Received 26 November 2020 Accepted 01 July 2021

Check for updates

(c) Author(s) (or their employer(s)) 2021. Re-use permitted under CC BY-NC. No commercial re-use. See rights and permissions. Published by BMJ.

\section{${ }^{1}$ Clinical and Academic} Department of Ophthalmology, Great Ormond Street Hospital For Children NHS Foundation Trust, London, UK

${ }^{2}$ School of Healthcare Science, Manchester Metropolitan University, Manchester, UK ${ }^{3} \mathrm{GOS}-\mathrm{ICH}$, University College London, London, UK

${ }^{4}$ Faculty of Science and Engineering, Manchester Metropolitan University, Manchester, UK

Correspondence to Oliver Rajesh Marmoy; o.marmoy@nhs.net

\section{ABSTRACT}

Introduction Raised intracranial pressure (rICP) can be a consequence of a variety of neurological disorders. A significant complication of rICP is visual impairment, due to retinal ganglion cell (RGC) dysfunction. In children, subjective measurements to monitor this, such as visual field examination, are challenging. Therefore, objective measurements offer promising alternatives for monitoring these effects. The photopic negative response (PhNR) is a component of the flash electroretinogram produced by RGCs; the cells directly affected in rICP-related vision loss. This project aims to assess the clinical feasibility and diagnostic efficacy of the PhNR in detecting and monitoring paediatric rICP.

Methods and analysis Section 1 is a cross-sectional study; group 1 young persons with disorders associated with rICP and a comparator group 2 of age-matched children without rICP. Both groups will undergo a PhNR recording alongside a series of structural and functional ophthalmic investigations, with the rICP group also having measurement of intracranial pressure.

Section 2 is a longitudinal study of the relationship between the PhNR and directly recorded intracranial pressure measurements, through repeated measures. PhNR amplitudes and peak times will be assessed against optical coherence tomography parameters, mean deviation of visual fields, other electrophysiology and ICP measurement through regression analyses.

Group differences between PhNR measurements in the rICP and control groups will be performed to determine clinically relevant cut-off values and calculation of diagnostic accuracy. Longitudinal analysis will assess PhNR amplitude against ICP measurements through regression analysis. Feasibility and efficacy will be measured through acceptability, practicality and sensitivity outcomes.

Ethics and dissemination Favourable opinion from a research ethics committee has been received and the study approved by Manchester Metropolitan University, the Health Research Authority and the Great Ormond Street Institute of Child Health (GOS-ICH) Research and Development office. This project is being undertaken as a doctoral award (ORM) with findings written for academic thesis submission, peer-reviewed journal and conference publications.
Strengths and limitations of this study

- This study investigates unique and objective methods of monitoring visual sequelae in childhood raised intracranial pressure (IICP) through the photopic negative response.

- Recruitment will involve both a control and a disease cohort, to improve our analysis of diagnostic sensitivity and feasibility of the photopic negative response (PhNR) testing in patients with rICP.

- All rICP group patient findings will be compared against measurements of intracranial pressure, which will allow direct analyses between indirect functional measurements and a gold-standard measure.

- The limitations of this study may include the current unknown variability of the PhNR, recorded to a modified protocol in children.

- One of the study outcome measures is visual field observation, which may not be possible in many children, therefore, analysis may rely on crossanalysis with optical coherence tomography imaging or intracranial pressure measurements alone.

\section{INTRODUCTION}

Raised intracranial pressure (rICP), synonymously termed intracranial hypertension ( $\mathrm{ICH})$, is a condition characterised by increased pressure within the cranium. The aetiology of rICP can be heterogeneous, typically resulting from a consequence of space occupying lesions, disturbance in cerebrospinal fluid (CSF) production or reabsorption, changes in blood volume, conditions affecting the cranial vault or alternatively can be idiopathic. ${ }^{1}$ In children, a number of disorders exist which can result in persistent rICP, such as craniosynostosis, idiopathic ICH (IIH) or hydrocephalus.

The symptoms of rICP can include chronic headache, vomiting, dizziness, tinnitus and visual dysfunction. The avoidance of visual 
impairment is one of the main objectives in the management of patients with rICP. As such, visual impairment is one of the most marked morbidities in IIH, sometimes up to $25 \% .^{2-4}$ Direct monitoring of ICP is an invasive procedure and poses risk of severe complications. ${ }^{5}$ In addition, the symptoms reported such as headache can be a heterogeneous or difficult feature for children to express. Therefore, monitoring the visual sequelae of rICP indirectly is critical in the diagnosis and monitoring of rICP, alongside providing functional information of vision to potentially reduce the risk of unnecessary neurosurgical monitoring in the paediatric population. Furthermore, the ability to objectively monitor visual function as a means to measure efficacy following neurosurgical intervention are also of benefit where structural changes may lack sensitivity or reactivity to improved ICP.

The CSF is continuous with the perioptic nerve sheath. Therefore, rICP produces physiological stress on the optic nerve and can give rise to axonal swelling, which can clinically be observed as 'papilloedema' or 'optic disc oedema'. ${ }^{6}$ In addition, rICP in children can cause additional postretinal changes to the visual pathway at the optic chiasm, optic radiations or occipital cortex, through dilation of the third or lateral ventricles which can compress or prolong the pathway tracts. ${ }^{7}$ The pathophysiology of visual loss in rICP is most predominantly result of retinal ganglion cell (RGC) dysfunction, secondary to mechanical pressure from the optic nerve sheath disrupting axoplasmic flow, causing papilloedema related visual field (VF) defects such as peripheral field constriction, enlarged blind spot or inferonasal stepping. ${ }^{9}$ Effective methods for monitoring visual dysfunction from rICP is unfortunately far more challenging in children than adults due to lower cooperation or ability to perform subjective testing, such as with VF examination. Effective objective methods are therefore essential to minimise the risk of irreversible visual impairment. ${ }^{5}$

Current consensus guidelines for adults with IIH suggests serial observation of papilloedema, visual acuity (VA) and VF assessment. ${ }^{10}$ However, both VA and papilloedema grading are controversially insensitive to change in rICP, with VF techniques being particularly challenging in children. ${ }^{11}$ Although some studies have demonstrated a positive correlation between the degree of VA impairment to lumbar puncture (LP) opening pressure in rICP, ${ }^{12}$ others have shown that VA remains normal despite direct evidence of rICP, and only visual evoked potentials (VEPs) have revealed an abnormality. ${ }^{13}$ Perhaps most sensitive to rICP are VF measurements, however, these are inherently challenging in children as they demand stable fixation, subjective reaction and alertness for a long period of time. ${ }^{3}$

An alternative method of assessing the effects of raised ICP on visual pathway function objectively in children of all ages is through visual electrophysiology testing. Visual electrophysiology and the pattern VEP in particular have been used to monitor visual compromise in children at risk of rICP, and previous publications from
Great Ormond Street Hospital (GOSH) have shown the VEP to have higher sensitivity than optic disc appearance and VA alone. ${ }^{13} 14$ Recent evidence suggests the pattern electroretinogram (PERG) and the photopic negative response (PhNR) of the full-field ERG that monitor RGC function may be valuable markers of optic nerve compromise in glaucoma and adult IIH cases. ${ }^{15}{ }^{16}$ However, the PERG demands corneal electrodes, high levels of patient cooperation and tests only the macula, which may remain unaffected until the late/advanced disease stages and is perhaps insensitive to peripheral VF constriction associated with raised ICP. ${ }^{17}$ In contrast, the PhNR records the global RGC response, recorded from photopic red flashes on a blue background to a full-field stimulus. Skin electrodes can be used and are much better tolerated by children than corneal electrodes, with the PhNR having the advantage of not demanding any prolonged or strict fixation (which the PERG does). In addition, we hypothesise that the PhNR as a full-field response will be more sensitive to the visual-field related changes observed in rICP than VA or other electrophysiological techniques, and therefore, may serve as a sensitive objective tool for the detection of RGC dysfunction and its relation to rICP in paediatric patients.

This project protocol is a cross-sectional and longitudinal study whereby all patients referred with a provisional or known diagnosis of IIH, hydrocephalus or craniosynostosis will be recruited. Patients will undergo routine clinical visual electrophysiology tests that will additionally include a PhNR protocol. The clinical benefit of the PhNR in the monitoring of visual dysfunction in patients with rICP will be compared with VA, ophthalmic imaging, $\mathrm{VF}$ and ultrasound measurements of the optic nerve, to ascertain the relationship between these measurements and the PhNR as a measurement of global RGC function. Additional analysis will assess the relationship between ICP and the PhNR in those patients whom undergo ICP measurements.

\section{METHODS AND ANALYSIS}

This study is based on a cross-sectional design with an additional longitudinal arm for patients undergoing direct ICP monitoring. All participants in the disease group will be children presenting to the service with IIH, craniosynostosis or hydrocephalus. Those in the control group will be patients seen for testing within the ophthalmology department and found to have normal ophthalmic examination, or with conditions unknown to have a relationship with rICP.

\section{Primary objective}

Evaluate the feasibility and clinical efficacy of the PhNR in conditions of rICP (IIH, craniosynostosis, hydrocephalus) and the relationship to other functional (VEP, VA, VF), structural (Optical Coherence Tomography, OCT, fundus photographs) and clinical (ICP, headaches) measures, to determine a clinical reference ranges and 


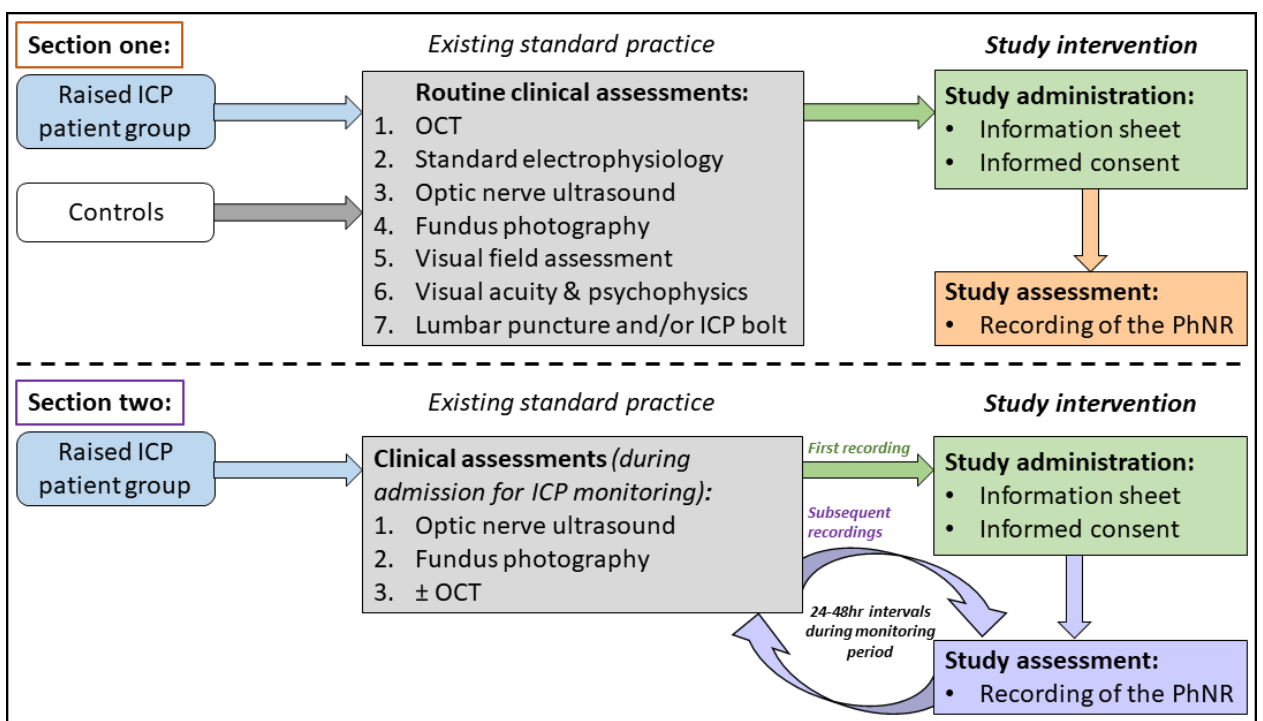

Figure 1 Study design. Section 1 demonstrates that disease and control groups will undergo routine ophthalmic examination as per standard practice, followed by recruitment procedures and recording of the photopic negative response (PhNR). Section 2 demonstrates the disease group undergoing intracranial bolt monitoring, will undergo standard clinical measurements during the monitoring period, followed by recruitment procedures. The PhNR will then be recorded at 24-48hours intervals during the monitoring period, up to a maximum of 14 days. ICP, intracranial pressure; OCT, optical coherence tomography.

efficacy of the PhNR for the longitudinal monitoring of patients with suspected or known rICP.

\section{Secondary objective/s}

- Assess whether the PhNR has independent power and sensitivity for the detection of rICP compared with a 'healthy' control group where there are no other imaging, electrophysiological or clinical correlates of retinal or intracranial pathology.

- Compare direct longitudinal measures of ICP to $\mathrm{PhNR}$ response amplitude to assess any relationship between RGC dysfunction with ICP changes.

- Assess the feasibility of implementing the PhNR in clinical practice as a diagnostic technique.

\section{Study design}

This study is divided into two primary research sections (figure 1).

Section 1: Participants with a provisional or known diagnosis of IIH, hydrocephalus or craniosynostosis, will form the 'disease' group and undergo PhNR testing. A healthy control group will be recruited from patients who have normal ophthalmic examination and no conditions associated with rICP. PhNR testing will be performed alongside standard clinical diagnostic tests to include other visual electrophysiology, ophthalmic imaging (OCT, fundus imaging), psychophysics (VA, VF, contrast and colour vision) and clinical examination/history taking. The aim of this section is to ascertain the discriminatory ability of the PhNR to distinguish between disease and healthy groups, and the relationship between the PhNR to other ophthalmic measurements (ie, OCT). Furthermore, in patients in the disease group, those patients undergoing ICP or LP measurements will have their respective ICP level noted and PhNR recorded close to this time to ascertain the direct relationship between ICP and the PhNR.

Section2: Patients undergoing intracranial measurement of ICP will have PhNR recorded longitudinally at 24-48 hour intervals to assess the relationship between ICP and PhNR over time.

\section{Study setting}

This is a single-centre study taking place at GOSH for Children National Health Service (NHS) Foundation Trust.

\section{Eligibility criteria \\ Recruitment}

Section 1: Participants will be screened for eligibility through referrals made to the Clinical and Academic Department of Ophthalmology at GOSH. Eligible participants will be provided a participant information sheet and written consent obtained if the invitation to participate is accepted. The disease group will be those patients with a primary diagnosis within the inclusion criterion, the control group will be those patients whom have normal ophthalmic examination through standard clinical measures without conditions known to cause rICP (table 1). Best clinical efforts will be made for the PhNR to be recorded as close to the date of the ICP bolt or LP measurement where performed in the disease group. Once test procedures are complete, data will be anonymised and collated for analysis.

Section 2: The principal investigator will liaise with specialist neuro-ophthalmology, neurology and neurosurgical teams to identify patients whom are undergoing investigation for rICP. In addition, those on planned lists for being monitored directly for rICP (ie, through a subarachnoid bolt or intraparenchymal monitor) will be 
Table 1 Inclusion and exclusion criteria for recruitment

\begin{tabular}{|c|c|c|c|}
\hline Section 1-inclusion criteria & Section 1-exclusion criteria & Section 2-inclusion criteria & Section 2-exclusion criteria \\
\hline $\begin{array}{l}\text { Patients between the ages } \\
\text { of } 1-16 \text { years old, should the } \\
\text { patient be cooperative enough } \\
\text { to tolerate testing }\end{array}$ & $\begin{array}{l}\text { Patient has significant } \\
\text { comorbid disease which could } \\
\text { significantly influence visual } \\
\text { electrophysiology interpretation, } \\
\text { outside of that expected for } \\
\text { raised ICP (ie, significant retinal } \\
\text { dysfunction). }\end{array}$ & $\begin{array}{l}\text { Patients between the ages } \\
\text { of } 1 \text { and } 16 \text { years, who are } \\
\text { undergoing ICP monitoring } \\
\text { within GOSH }\end{array}$ & $\begin{array}{l}\text { Patient has comorbid disease } \\
\text { which may affect study findings }\end{array}$ \\
\hline $\begin{array}{l}\text { For the control group: patients } \\
\text { with otherwise normal visual } \\
\text { electrophysiology and no } \\
\text { objective evidence of retinal or } \\
\text { optic nerve disease or condition } \\
\text { known to cause rICP }\end{array}$ & $\begin{array}{l}\text { Patient has symptomatic cause } \\
\text { for raised ICP which could } \\
\text { confound test findings (ie, frontal } \\
\text { lobe meningioma or other space } \\
\text { occupying lesions affecting the } \\
\text { visual pathway) }\end{array}$ & $\begin{array}{l}\text { Participants consent to serial } \\
\text { recording of PhNR }\end{array}$ & \\
\hline $\begin{array}{l}\text { Fundus imaging or OCT } \\
\text { performed as per standard } \\
\text { practice }\end{array}$ & \multirow{2}{*}{$\begin{array}{l}\text { Patient is on sedative or other } \\
\text { medication which has a potential } \\
\text { but unknown effect on the PhNR } \\
\text { component of the ERG. }\end{array}$} & $\begin{array}{l}\text { Patient is alert or in natural sleep } \\
\text { for testing during ICP monitoring } \\
\text { period }\end{array}$ & \\
\hline $\begin{array}{l}\text { Patient must be referred for } \\
\text { visual electrophysiology testing } \\
\text { as per standard practice (ie, } \\
\text { recruited participants will only } \\
\text { be those standardly referred } \\
\text { to this service to maintain } \\
\text { standard practice) }\end{array}$ & & $\begin{array}{l}\text { Other causes for rICP will be } \\
\text { included, so long as they do } \\
\text { not knowingly affect the visual } \\
\text { pathways }\end{array}$ & \\
\hline
\end{tabular}

ERG, electroretinogram; GOSH, Great Ormond Street Hospital; ICP, intracranial pressure; IIH, idiopathic intracranial hypertension; OCT, optical coherence tomography; PhNR, photopic negative response; rICP, raised intracranial pressure.

identified and recruited with written consent obtained if the invitation to participate is accepted.

\section{Consent}

Age-appropriate research ethics committee (REC) approved information sheets have been created for ages 2-5, 6-11 and 12-15 and 16+ years old and for parents/ guardians. If the patient and family (parent/guardian) are happy to proceed onto the study an assent or consent form will be completed by the parent/guardian and/or patient where deemed gillick competent. Patients and their families will be offered the opportunity to put questions to the research team before informed consent/ assent is taken. Patients and their families are free to withdraw consent at any time during the study period (including monitoring period). For section 2, it is emphasised that although repeat measures of PhNR over the monitoring period are planned, participants may stop PhNR testing at any time.

\section{Study procedures}

Patients within both disease and control groups will undergo routine clinical 'standard' testing to measure structural and functional ophthalmic characteristics (figure 1). In addition to standard visual electrophysiology tests (ERG, VEP and PERG), for section 1 the patient will also have their PhNR recorded as an addition to standard practice, taking approximately an additional 5-10 min of clinical time. Only those patients in the disease group will have their ICP or LP data collected for inclusion as those in the control group will not have their ICPs monitored. For section 2, while the patient is undergoing ICP monitoring, PhNR measurements will be sampled at 24-48 hours intervals for the duration of ICP monitoring.

\section{Recording the PhNR}

A modified paediatric protocol has been developed by the authors for the measurement of the PhNR in children. This deviates from the extended international protocol which prefers corneal electrodes, pupil dilation and a ganzfeld bowl stimulus which can make the recording more challenging for children. ${ }^{18}$ Our protocol, in brief, uses skin electrodes with an active electrode placed on the central lower lid and reference electrode placed at the ipsilateral outer canthus, as used within our routine paediatric ERG protocol. ${ }^{19} 20$ A ground electrode is typically placed on the forehead.

The stimulus delivered is a red flash $(\sim 630 \mathrm{~nm},<5 \mathrm{~ms}$ duration) on a rod-saturating steady blue background $\left(\sim 455 \mathrm{~nm}\right.$, minimum $\sim 10$ photopic cd. $\left.\mathrm{m}^{2}\right)$ at $3-5$ flashes 
per second. The flash strength will range between 0.3 and $3.9 \mathrm{log}$ photopic cd.s $/ \mathrm{m}^{2}$ to model the PhNR intensityresponse function. The flash stimuli will be delivered from a new handheld flash LED stimulator capable of delivering chromatic flashes (ColorFlash, Diagnosys (Lowell, Massachusetts, USA)) binocularly, with flashes delivered within $10 \mathrm{~cm}$ from the eyes. Signals will be averaged and a minimum of two averages will be obtained with 100 trials within each, with a minimum of 10 trials used as a standard, though this may be dependent on patient cooperation. Filter settings will be set to $0.3-300 \mathrm{~Hz}$.

\section{Standard clinical measurements}

\section{Routine visual electrophysiology testing}

Pattern and flash VEP results will be incorporated into the analysis by recording the amplitude and peak-time of the major positive peaks elicited to a range of check widths and/or flashes. The amplitude of the PERG N95 component and the ratio of N95:P50 amplitudes will be included. All responses will be recorded in accordance with ISCEV standards. ${ }^{21} 22$

\section{Optical coherence tomoography, fundus photography and ultrasound of the optic nerve}

Spectralis OCT will be performed by an appropriately trained staff member as per current practice. The neuroretinal rim will be measured to visualise the axial cross-section of the optic nerve head alongside the peripapilliary retinal nerve fibre layer (RNFL) thickness. Additional measures of Ganglion Cell Complex volume (GCCV) will be measured from scans of the macula (exceeding $20^{\circ} \times 20^{\circ}$ ) measuring the boundary between the inner plexiform layer and inner nuclear layer. Colour fundus photography will be collected using non-mydriatic photography, either through Optomap (Optos, Optos PLC, Dumferline) or a hand-held fundus camera. Ultrasonic examination of the optic nerve will include the identification of optic nerve drusen, and the perioptic nerve sheath diameter recorded in lateral gaze as per current practice.

\section{VA and psychophysical testing}

VA, contrast sensitivity and colour vision measurements will be performed. VFs will ideally be performed using a 30-2 SITA technique with kinetic isopters for peripheral field examination, or in less cooperative children a kinetic VF will be prioritised (Octopus 900, Haag-Streit, Switzerland).

\section{Intracranial pressure measurements}

The measurements of ICP will be obtained as part of standard clinical practice through two means: (1) from clinically obtained Lumbar Puncture opening pressures measured as centimeters of the water column $\left(\mathrm{cm} \mathrm{H}_{2} 0\right)$ from patients in the lateral decubitus position. Pressures will be considered 'equivocal' between 25 and $28 \mathrm{~cm}$ $\mathrm{H}_{2} 0$ and 'raised' in those exceeding $28 \mathrm{~cm} \mathrm{H}_{2} 0^{23}$ and (2) Through direct ICP measurements using an intraparenchymal fiberoptic ICP monitor or subdural bolt monitor.
In line with ${ }^{24}$ and subsequent corroborating literature, an ICP between 10 and $15 \mathrm{~mm} \mathrm{Hg}$ will be considered borderline, with those exceeding $15 \mathrm{~mm} \mathrm{Hg}$ considered raised. However, within this centre rICP is typically considered through a multidiscipilinary team meeting following a 48 hours ICP bolt recording to review the entire ICP trace, including mean amplitude, RAP index and ICP relationship to sleep stage ${ }^{2425}$ alongside the childs wellness and circumstance. Therefore, while the criteria for mean amplitude will be used, the outcome from the Neurosurgical MDT will be used to determine rICP to incorporate other indices of rICP (ie, RAP index or ICP spikes).

\section{Feasibility testing}

Clinical feasibility will be measured by acceptability of the PhNR test to patients in terms of the number of successful recordings in children of all ages relative to the number of attempts. Practicality of the PhNR will also be explored, which will include analysis of average time taken for the test and its value over existing practice in the detection of abnormalities further to that already identified within routine testing.

\section{Data analysis}

Section 1 is a cross-sectional study design. A priori power analysis using a power of 0.80 and alpha of 0.05 based on published PhNR amplitude effect changes in glaucomatous eyes was made (mean $\pm \mathrm{SEM}$ ) was $30.67 \mu \mathrm{V} \pm 10.02 \mu \mathrm{V}$ in the 'normal' group and $15.83 \mu \mathrm{V} \pm 8.18 \mu \mathrm{V}$ in the glaucoma group, ${ }^{26}$ which predicts with a two-tailed hypothesis that 41 samples are required per group for statistical significance. Therefore a total of 60 participants within the disease group are required to allow $\pm 30 \%$ for spurious, poor quality or excluded data. The control group will be age and sample size matched for direct comparison of these groups.

Section 2 is a longitudinal within-subject design. The PhNR amplitude will be compared with directly recorded ICP monitor outputs. The time-course of these changes will be assessed using regression analysis to discern whether the PhNR component is sensitive to ICP changes directly. This use will allow us to indirectly validate the PhNR component in monitoring of patients ICP changes in the outpatient setting where direct monitoring is unachievable. This direct comparison potentially will allow us to establish a 'limit' for abnormal PhNR which may warrant future therapeutic intervention in the outpatient setting alongside OCT measurements of papilloedema.

The photopic flash ERG waveform comprises of the a-wave, b-wave, i-wave and PhNR (figure 2). As there is variation regarding the optimal measurement methods for the PhNR, the amplitude will be measured from both the baseline to the trough alongside the peak of the b-wave to the trough, with peak-times all recorded, respectively. Post hoc calculation of PhNR:b-wave ratio will be calculated to ensure that any potential b-wave reduction does not falsely cause inaccurate interpretation of PhNR amplitude loss. Statistical treatment of data will determine 


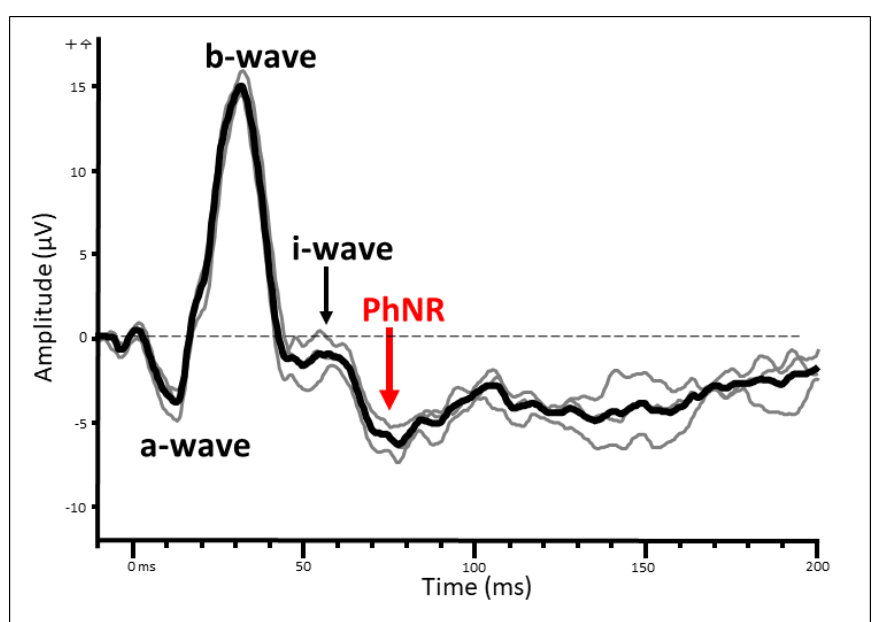

Figure 2 A representative photopic negative response (PhNR) waveform in a healthy adult participant. The ERG response is seen as the a-wave and b-wave, respectively, followed by the negative waveform following the b-wave and i-wave known as the PhNR, which is the measure of interest within this study. This waveform is that typically observed when recording the PhNR to this studies modified PhNR protocol, typical b-wave amplitudes within 10-30 uV.

a clinically relevant 'cut-off' for determining an abnormal PhNR amplitude through examination of normal PhNR and abnormal PhNR related to other outcome measures. However, previous studies would suggest a cut-off of between $30 \%$ and $50 \%$ below the lower reference limit is reasonable. ${ }^{1626-29}$

Comparative clinical measurements will be related to PhNR findings to assess the relationship between structural (OCT, ultrasound), functional (visual acuity (VA), routine visual electrophysiology psychophysics) and neurological (LP/bolt ICP) changes associated with PhNR findings. Previous studies indicate the most common findings in papilloedema in Craniosynostosis are increased Total Retinal Thickness, Retinal Nerve Fibre Thickness, central optic disc thickness and maximal anterior retinal projection, which will be used within our analyses. ${ }^{30} \mathrm{B1} \mathrm{VF}$ mean deviation will be measured alongside optic nerve sheath diameter measurement against laboratory reference values. If normally distributed, data will be analysed using analysis of variance or independent two-tailed t-tests to determine any statistical differences between PVEP amplitude and latency, PhNR amplitude/ latency and RNFL/GCCV thickness' in OCT imaging. A Benjamini-Hochberg post hoc correction will be made to allow for multiple analyses. Additionally, receiver operating characteristics will be determined to determine a potential 'cut-off' between disease and control groups. The association between structural, functional and PhNR measurements may also be studied with multiple regression analyses or Pearson correlation coefficients, constructed using the $\log$ PhNR and RNFL thickness or GCCV thickness as the independent variables. These will be assessed for their fit, demonstrated through the correlation coefficient $(r)$ and statistical significance of the coefficient to identify the relationship between PhNR and standard clinical variables.

Feasibility will be calculated using qualitative perspectives from the staff at GOSH alongside recording the time taken for each PhNR recording, the number of successful attempts relative to the total number of events, quality of data (calculated by signal-to-noise).

\section{Patient and public involvement}

No patients were involved in the structure or design of this research. We plan to hold patient involvement groups for dissemination of our findings to participants and patients of the research.

\section{DISCUSSION}

We describe the protocol for our prospective study examining the PhNR in the diagnosis and monitoring of children with rICP. Our proposed methodology incorporates a study design, which uses measurements of ICP against the PhNR among other ophthalmological tests. In this design, we hope to recruit the majority of patients from those undergoing direct ICP monitoring, however, in practice it may be likely that an equal number of patients will have their ICP status determined from the less direct LP opening pressure. While correction values can be applied to these two measurements to make their output comparable, their reference values within the cited literature and within clinical practice are not directly comparable. We have, therefore, opted to determine rICP status independently for each method. In this way, we can independently determine rICP in patients for group analysis which should minimise the risk of biasing the sample, however, for assessing relationship between variables (ie, when fitting statistical models for PhNR amplitude vs ICP) we may have to apply correction factors to make ICP an interval scale.

Section 1 of our protocol describes sampling from a cross-sectional study design. Therefore, while we can assess the relationship between the PhNR, ICP and ophthalmic measures at one time point, this methodology is limited in providing strong evidence of a causal or temporal relationship between these variables. This prompted the need for section 2 of our study, which assesses the longitudinal changes of the PhNR related to ICP, so we can examine the temporal relationship between these variables of interest. Furthermore, while section 1 is a crosssectional design, including a control group will inform us of clinical decision limits and the clinical efficacy of the $\mathrm{PhNR}$ in practice for discriminating between normal and rICP groups.

Ophthalmic examination in paediatrics can sometimes be challenging, in particular when attempting detailed ophthalmic imaging or electrophysiology testing. We anticipate that a minority of young or less cooperative patients may be unable to cooperate with all clinical and study testing, resulting in incomplete datasets. This was considered within the study methodology, where 
it was decided we would need to examine the relationship between independent variables, rather than purely observe mixed effects. Furthermore, we do not yet know the variability of the PhNR in children, which we hope to establish from data within our control group. The logistics surrounding recruitment are likely to be somewhat challenging, as we would opt for patients in section 1 to have their PhNR assessments as close as possible to their ICP measurement, but this may not always be possible. Once recruitment has started, if this appears to be challenging we may need to determine a time period whereby recruitment would not be beneficial for the study outcomes.

While the underpinning hypothesis is addressed within this study, there are some factors for which current technology limits our ability to attribute causal relationships; most particularly for OCT measurements. The PhNR is a response reflecting global RGC activity, however, within current clinically available technology we are only able to examine the GCCV around the central $30^{\circ}$, alongside the RNFL. It may be that there is a poor relationship between the central GCCV and PhNR in rICP, given that the VF complaints are predominantly in the visual periphery. We attempt to compensate for this limitation by including peripheral VF assessments for our cohorts, to provide some indices of peripheral field functionality (ie, as an indirect reflection of global RGC sensitivity/function) to complement the perhaps limited OCT spatial value.

This study, to our knowledge, is the first to examine the PhNR in children with rICP. We are optimistic that this may prove to be a valuable tool in assessing and monitoring children with rICP, alongside providing discriminatory ability against other conditions of the optic nerve or RGCs. Our technique for recording the PhNR is noninvasive and does not require pupil dilation, making it acceptable to children of all ages and cooperation levels.

\section{ETHICS AND DISSEMINATION}

This study has received favourable NHS REC opinion (reference 18/LO/1035) in 2018 alongside HRA approval and research and development office approvals at the research site and from the sponsor.

We anticipate the findings of this study will direct future visual electrophysiology monitoring of patients with conditions of raised ICP alongside validation and reflection on current practices, in order to better guide treatment of these patient groups.

The findings will form a part of the PIs doctoral thesis examining visual monitoring in disorders of rICP, which is to be submitted to Manchester Metropolitan University in partial fulfilment of the Doctor of Clinical Science programme as part of the Higher Specialist Scientific Training Programme. The anonymised data may also be written for publication in a peer-reviewed journal or conference presentation.

Twitter Oliver Rajesh Marmoy @0_Marmoy
Contributors ORM conceived of the presented hypothesis. ORM, DAT and EH-T contributed to the design of the study protocol and proposed acquisition and data analysis strategy. ORM, DAT and EH-T will provide interpretation of study data. ORM drafted the manuscript first draft with significant contributions from DAT and EH-T prior to submission. All authors approve of the final version of this manuscript and agree collective accountability for all aspects of this work.

Funding This work is supported by Health Education England as part of the Higher Specialist Scientific Training programme. The annual bursary received has supported this research and in part fulfilment of the DClinSci at Manchester Metropolitan University (OM).

Competing interests None declared.

Patient and public involvement Patients and/or the public were not involved in the design, or conduct, or reporting, or dissemination plans of this research.

Patient consent for publication Not required.

Provenance and peer review Not commissioned; externally peer reviewed.

Open access This is an open access article distributed in accordance with the Creative Commons Attribution Non Commercial (CC BY-NC 4.0) license, which permits others to distribute, remix, adapt, build upon this work non-commercially, and license their derivative works on different terms, provided the original work is properly cited, appropriate credit is given, any changes made indicated, and the use is non-commercial. See: http://creativecommons.org/licenses/by-nc/4.0/.

ORCID iD

Oliver Rajesh Marmoy http://orcid.org/0000-0003-2022-3669

\section{REFERENCES}

1 Dunn LT. Raised intracranial pressure. J Neurol Neurosurg Psychiatry 2002;73 Suppl 1:i23-7.

2 Corbett JJ, Savino PJ, Thompson HS, et al. Visual loss in pseudotumor cerebri. follow-up of 57 patients from five to 41 years and a profile of 14 patients with permanent severe visual loss. Arch Neurol 1982;39:461-74.

3 Rowe FJ, Sarkies NJ. Assessment of visual function in idiopathic intracranial hypertension: a prospective study. Eye 1998;12:111-8.

4 Rowe FJ. Assessment of visual function in idiopathic intracranial hypertension. Br J Neurosurg 2011;25:45-54.

5 Wiegand C, Richards P. Measurement of intracranial pressure in children: a critical review of current methods. Dev Med Child Neurol 2007;49:935-41.

6 Tso MO, Hayreh SS. Optic disc edema in raised intracranial pressure. IV. axoplasmic transport in experimental papilledema. Arch Ophthalmol 1977;95:1458

7 Humphrey PR, Moseley IF, Russell RW. Visual field defects in obstructive hydrocephalus. J Neurol Neurosurg Psychiatry 1982;45:591-7.

8 Menjot de Champfleur N, Menjot de Champfleur S, Galanaud D, et al. Imaging of the optic chiasm and retrochiasmal visual pathways. Diagn Interv Imaging 2013;94:957-71.

9 Hayreh SS. Pathogenesis of optic disc edema in raised intracranial pressure. Prog Retin Eye Res 2016;50:108-44.

10 Mollan SP, Hornby C, Mitchell J, et al. Evaluation and management of adult idiopathic intracranial hypertension. Pract Neurol 2018;18:485-8

11 Tuite GF, Chong WK, Evanson J, et al. The effectiveness of papilledema as an indicator of raised intracranial pressure in children with craniosynostosis. Neurosurgery 1996;38:272-8.

12 Ding J, Zhou D, Geng T, et al. To predict visual deterioration according to the degree of intracranial hypertension in patients with cerebral venous sinus thrombosis. Eur Neurol 2018;80:28-33.

13 Liasis A, Nischal KK, Walters B, et al. Monitoring visual function in children with syndromic craniosynostosis: a comparison of 3 methods. Arch Ophthalmol 2006;124:1119-26.

14 Thompson DA, Liasis A, Hardy S, et al. Prevalence of abnormal pattern reversal visual evoked potentials in craniosynostosis. Plast Reconstr Surg 2006;118:184-92.

15 Kim HD, Park JY, Ohn Y-H. Clinical applications of photopic negative response (PhNR) for the treatment of glaucoma and diabetic retinopathy. Korean Journal of Ophthalmology 2010;24:89-95.

16 Moss HE, Park JC, McAnany JJ. The photopic negative response in idiopathic intracranial hypertension. Invest Ophthalmol Vis Sci 2015;56:3709-14.

17 Park JC, Moss HE, McAnany JJ. Electroretinography in idiopathic intracranial hypertension: comparison of the pattern ERG and the photopic negative response. Doc Ophthalmol 2018;136:45-55. 
18 Frishman L, Sustar M, Kremers J, et al. ISCEV extended protocol for the photopic negative response (PhNR) of the full-field electroretinogram. Doc Ophthalmol 2018;136:207-11.

19 Kriss A, Russell-Eggitt I. Electrophysiological assessment of visual pathway function in infants. Eye 1992;6:145-53.

20 Marmoy OR, Moinuddin M, Thompson DA. An alternative electroretinography protocol for children: a study of diagnostic agreement and accuracy relative to ISCEV standard electroretinograms. Acta Ophthalmol 2021. doi:10.1111/aos.14938. [Epub ahead of print: 14 Jun 2021].

21 Bach M, Brigell MG, Hawlina M, et al. ISCEV standard for clinical pattern electroretinography (PERG): 2012 update. Doc Ophthalmol 2013;126:1-7.

22 Odom JV, Bach M, Brigell M, et al. ISCEV standard for clinical visual evoked potentials: (2016 update). Doc Ophthalmol 2016;133:1-9.

23 Avery RA, Shah SS, Licht DJ, et al. Reference range for cerebrospinal fluid opening pressure in children. $N$ Engl J Med 2010;363:891-3.

24 Renier D, Sainte-Rose C, Marchac D, et al. Intracranial pressure in craniostenosis. J Neurosurg 1982;57:pp.Vol:370-7. Vol.
25 Hayward R, Britto J, Dunaway D, et al. Connecting raised intracranial pressure and cognitive delay in craniosynostosis: many assumptions, little evidence. J Neurosurg Pediatr 2016;18:242-50.

26 Kim HD, Park JY, Ohn Y-H. Clinical applications of photopic negative response (PhNR) for the treatment of glaucoma and diabetic retinopathy. Korean J Ophthalmol 2010;24:89-95.

27 Gotoh Y, Machida S, Tazawa Y. Selective loss of the photopic negative response in patients with optic nerve atrophy. Arch Ophthalmol 2004;122:341-6.

28 Wu Z, Hadoux X, Fan Gaskin JC, et al. Measuring the photopic negative response: viability of skin electrodes and variability across disease severities in glaucoma. Trans/ Vis Sci Technol 2016;5:13-19.

29 Joshi NR, Ly E, Viswanathan S. Intensity response function of the photopic negative response (PhNR): effect of age and test-retest reliability. Doc Ophthalmol 2017;135:1-16.

30 Driessen C, Eveleens J, Bleyen I, et al. Optical coherence tomography: a quantitative tool to screen for papilledema in craniosynostosis. Childs Nerv Syst 2014;21:1067-73.

31 Swanson JW, Aleman TS, Xu W, et al. Evaluation of optical coherence tomography to detect elevated intracranial pressure in children. JAMA Ophthalmol 2017;135:320-8. 